\title{
Machine Learning-Based Identification of Suicidal Risk in Patients With Schizophrenia Using Multi-Level Resting-State fMRI Features
}

\begin{abstract}
Bartosz Bohaterewicz ${ }^{1,2 *}$, Anna M. Sobczak ${ }^{1 *}$, Igor Podolak ${ }^{3}$, Bartosz Wójcik³, Dagmara Mętel ${ }^{4}$, Adrian A. Chrobak ${ }^{5}$, Magdalena Fąfrowicz ${ }^{1}$, Marcin Siwek ${ }^{6}$, Dominika Dudek ${ }^{5}$ and Tadeusz Marek ${ }^{1}$
\end{abstract}

'Department of Cognitive Neuroscience and Neuroergonomics, Institute of Applied Psychology, Jagiellonian University, Kraków, Poland, ${ }^{2}$ Department of Psychology of Individual Differences, Psychological Diagnosis, and Psychometrics, Institute of Psychology, University of Social Sciences and Humanities, Warsaw, Poland, ${ }^{3}$ Institute of Computer Science, Faculty of Mathematics and Computer Science, Jagiellonian University, Kraków, Poland, ${ }^{4}$ Department of Community Psychiatry, Jagiellonian University Medical College, Kraków, Poland, ${ }^{5}$ Department of Adult Psychiatry, Jagiellonian University Medical College, Kraków, Poland, ${ }^{6}$ Department of Affective Disorders, Jagiellonian University Medical College, Kraków, Poland

OPEN ACCESS

Edited by:

Qiuyun Fan,

Harvard Medical School, United States

Reviewed by:

Xize Jia,

Hangzhou Normal University, China

Rosaleena Mohanty,

Karolinska Institutet (KI), Sweden

*Correspondence:

Anna M. Sobczak

ann.marie.sobczak@gmail.com

Bartosz Bohaterewicz

bohaterewicz@gmail.com

Specialty section:

This article was submitted to Brain Imaging Methods, a section of the journal

Frontiers in Neuroscience

Received: 12 September 2020

Accepted: 26 November 2020

Published: 11 January 2021

Citation:

Bohaterewicz B, Sobczak AM,

Podolak I, Wójcik B, Mẹtel D, Chrobak AA, Fąfrowicz M, Siwek M, Dudek D and Marek T (2021) Machine

Learning-Based Identification of Suicidal Risk in Patients With Schizophrenia Using Multi-Leve

Resting-State fMRI Features.

Front. Neurosci. 14:605697. doi: 10.3389/fnins.2020.605697
Background: Some studies suggest that as much as $40 \%$ of all causes of death in a group of patients with schizophrenia can be attributed to suicides and compared with the general population, patients with schizophrenia have an 8.5-fold greater suicide risk (SR). There is a vital need for accurate and reliable methods to predict the SR among patients with schizophrenia based on biological measures. However, it is unknown whether the suicidal risk in schizophrenia can be related to alterations in spontaneous brain activity, or if the resting-state functional magnetic resonance imaging (rsfMRI) measures can be used alongside machine learning $(\mathrm{ML})$ algorithms in order to identify patients with SR.

Methods: Fifty-nine participants including patients with schizophrenia with and without SR as well as age and gender-matched healthy underwent 13 min resting-state functional magnetic resonance imaging. Both static and dynamic indexes of the amplitude of low-frequency fluctuation (ALFF), the fractional amplitude of low-frequency fluctuations (fALFF), regional homogeneity as well as functional connectivity (FC) were calculated and used as an input for five machine learning algorithms: Gradient boosting (GB), LASSO, Logistic Regression (LR), Random Forest and Support Vector Machine.

Results: All groups revealed different intra-network functional connectivity in ventral DMN and anterior SN. The best performance was reached for the LASSO applied to FC with an accuracy of $70 \%$ and AUROC of $0.76(p<0.05)$. Significant classification ability was also reached for GB and LR using fALFF and ALFF measures.

Conclusion: Our findings suggest that SR in schizophrenia can be seen on the level of DMN and SN functional connectivity alterations. ML algorithms were able to significantly differentiate SR patients. Our results could be useful in developing neuromarkers of SR in schizophrenia based on non-invasive rsfMRI.

Keywords: schizophrenia, suicidal ideations, machine learning, resting state fMRI, mental pain, classification, gradient boosting, feature selection 


\section{INTRODUCTION}

Schizophrenia research suggests that as much as $40 \%$ of all death causes in this group can be attributed to suicides (Wildgust et al., 2010), while $25-50 \%$ of individuals with schizophrenia attempt to commit suicide during their lifetime (Bohaterewicz et al., 2018; Cassidy et al., 2018). Hence, there is a vital need of developing more accurate and objective methods to predict the risk of suicide among individuals with schizophrenia.

Functional magnetic resonance imaging (fMRI) is a noninvasive, widely employed method allowing one to measure activity of a human brain. Resting state (rs), in turn, is considered highly effective as it captures $60-80 \%$ of the brain's total activity (Smitha et al., 2017). Furthermore, some studies show that it allows monitoring treatment outcomes as well as assessing biomarkers of psychiatric disorders (Glover, 2011; Moghimi et al., 2018).

Previous studies indicate gray matter volume reduction in dorsolateral prefrontal cortex (DLPFC), superior temporal gyrus, as well as insular cortex in patients after suicide attempt, compared to the ones without suicide attempt in the past (Besteher et al., 2016; Zhang et al., 2020), whereas fMRI studies revealed that during a simple task based on cognitive control, suicide thoughts were associated with decreased activity in PFC and the history of previous suicide attempt resulted in decreased activity of premotor cortex (Minzenberg et al., 2014; Potvin et al., 2018). Previous results from volumetric as well as functional task fMRI analyses indicate the potential resting-state brain activity changes in the regions included in Default Mode Network (DMN), Salience Network (SN), and Sensorimotor Network (SMN).

In recent years, there has been a growing number of machine learning (ML) applications on rsfMRI data in order to make prognostic evaluation and to differentiate between various groups or conditions (Pereira et al., 2009). Lately, ML classifiers with the input from $\mathrm{fMRI}$ as an unbiased biomarker have been adopted to identify people engaged in the suicide-related behavior, including suicide ideations. For example, Just et al. (2017) were able to correctly identify 15 out of 17 suicidal participants with a sensitivity of 0.88 and a specificity of 0.94 using Gaussian Naïve Bayes algorithm and task fMRI data. In more recent work, Gosnell et al. (2019) used a Random Forest (RF) algorithm and rsfMRI functional connectivity data from psychiatric inpatients that enabled them to correctly classify suicidal behavior with a sensitivity of $81.3 \%$. To the best of our knowledge, none of the previous studies focused on various ML classifiers in order to discriminate between healthy controls (HCs), suicidal risk (SR), and non-suicidal risk (NSR) schizophrenia patients based on rsfMRI data.

In the current work, our objective was to conjoin ML methods with rsfMRI data in order to investigate whether the selected classifiers allow differentiating between schizophrenia patients with and without a suicide risk. Ultimately, five algorithms such as Gradient Boosting (GB), Least Absolute Shrinkage and Selection Operator (LASSO), Logistic Regression (LR), RF, and Support Vector Machine (SVM) were performed to increase the reliability of diagnostic accuracy. Each metric presents a different degree of complexity; therefore, establishing their separate and combined precision allows gaining a wider picture in the aforementioned classification. Moreover, the article aims at explaining the association between the resting-state brain activity and suicide risk among schizophrenia patients; thus, stationary as well as dynamic measures with sliding windows approach were calculated. Based on the literature, the authors hypothesize (a) varied neural activity in the regions included in DMN, SN, and SMN networks to be involved in suicide risk in patients with schizophrenia; (b) that the predictive ability of classifiers will be better while using dynamic indexes as the features in comparison to the static ones; (c) that the results of ML-based discrimination will differ using diverse parcellation approaches; (d) ML-based algorithms to discriminate between SR and NSR groups with the accuracy exceeding 50\%.

\section{MATERIALS AND METHODS}

\section{Participants}

A total of 66 participants were recruited for the study. The clinical group consisted of 43 patients with paranoid schizophrenia diagnosed according to the ICD-10 criteria. The clinical group covered two subgroups: 24 patients with acute suicidal risk (SR) and 19 patients without such risk (NSR). The control group (HCs) consisted of 23 individuals matched in terms of gender and age with a clinical group, without diagnosis of mental disorder or a history of mental illness in first-degree relatives. All the participants were righthanded, as measured by the Neurological Evaluation Scale (Buchanan and Heinrichs, 1989). The inclusion criterion for the clinical group was treatment with atypical antipsychotic agents from the group of dibenzoxazepine: clozapine, olanzapine, or quetiapine. Additionally, valproic acid treatment was accepted. The exclusion criteria for both clinical and control groups were as follows: (1) history of alcohol or drug abuse (according to substance use disorder of DSM-5); (2) severe, acute, or chronic neurological and somatic diseases; (3) severe personality disorders; (4) treatment other than those mentioned in the inclusion criteria. All of the abovementioned conditions were confirmed by clinical interviews based on DSM-5 criteria. Detailed information about patients' medication is presented in the Supplementary Materials. Written consent was obtained from all of the participants. The study was approved by the Jagiellonian University Bioethics Committee.

\section{Assessment of Suicidality}

Suicidal risk was assessed with the Polish adaptation of Suicide Behavior Questionnaire-Revised (SBQ-R) (Osman et al., 2001; Chodkiewicz and Gruszczyńska, 2020), with the cutoff of $\geq 8$ points in accordance with the Osman et al. (2001) recommendations. Moreover, the Polish adaptation (Chodkiewicz, 2013) of The Psychache Scale (TPS) (Holden et al., 2001) was used to evaluate the subjective experience of participants' psychological pain, considered to be highly associated with suicidal thoughts and acts (Ducasse et al., 2018). 


\section{MRI Data Acquisition}

MRI data were acquired using a 3-T Siemens Skyra MR System (Siemens Medical Solutions, Erlangen, Germany). Anatomical images were obtained using sagittal 3D T1-weighted MPRAGE sequence with $\mathrm{TR}=2,300 \mathrm{~ms}$ and $\mathrm{TE}=3.9 \mathrm{~ms}$. A total of 13 min of functional resting-state BOLD images was acquired using a gradient-echo single-shot echo planar imaging sequence with the following parameters: FOV $=256 \mathrm{~mm}$; TE $=27 \mathrm{~ms}$; $\mathrm{TR}=2060 \mathrm{~ms}$; voxel size $=3 \mathrm{~mm} \times 3 \mathrm{~mm} \times 3 \mathrm{~mm}$, with no gap. Altogether, 39 interleaved transverse slices and 400 volumes were acquired. During the resting-state procedure, the subjects were instructed to keep their eyes open and to think of nothing particular. They were also asked not to fall asleep, which was controlled using an infrared binocular eye tracker (Eyelink 1000 Plus, SR Research, Mississauga, ON, Canada). In addition, during the EPI sequence, neutral gray background was presented using a MRI-compatible LCD screen and Siemens Head Coil Viewing Mirror. Both structural and functional sequence details are in Supplementary Data Sheet 1.

\section{Data Preprocessing}

Data preprocessing was performed using Dpabi v. 4.2 (Yan et al., 2016) and SPM 12 (Friston, 1994), both working under Matlab v.2018a (The Mathworks Inc.). The first 10 time points were discarded due to signal equilibration, and next slice timing and realignment with assessment of the voxel specific head motion were conducted. The subjects with movements in one or more of the orthogonal directions above $3 \mathrm{~mm}$ or rotation above $3^{\circ}$ were discarded from the analysis. A total of three participants from the control group and four patients from the clinical sample (four from SR group) were excluded due to the excessive head movements. Consequently, 39 patients and $20 \mathrm{HCs}$ were included in the final analyses. Functional scans were then coregistered using T1 images and normalized to Montreal Neurological Institute (MNI) space using DARTEL and a voxel size of $3 \mathrm{~mm}^{3}$. The 24 motion parameters (Friston et al., 1996) derived from the realignment step were regressed out from the functional data by linear regression as well as five principal components from both cerebrospinal fluid and white matter signals using principal components analysis integrated in a Component-Based Noise Correction Method (Behzadi et al., 2007). The global signal was included due to its potential in providing additional valuable information (Liu et al., 2017). The signal was then band-pass filtered $(0.01-0.08 \mathrm{~Hz})$. Finally, the functional data were spatially smoothed with 4-mm Full Width at Half Maximum (FWHM) kernel.

\section{Parcellation}

For validation purposes and to exclude a chance of parcellationspecific results, the preprocessed data were parcellated using two functional atlases: Power et al. (2011), which utilizes 264 functionally independent regions, and Automated Anatomical Labeling (AAL) atlas, which separates brain into 116 regions (Tzourio-Mazoyer et al., 2002). Using centroids obtained from both atlases, the raw signal from individual brain maps was extracted and averaged within a 4-mm-radius sphere using
MarsBaR v. 0.43 (Brett et al., 2002). In addition, in accordance to our hypothesis, in order to investigate possible betweengroup differences among DMN, SN, and SM networks, the authors used templates from FIND lab ${ }^{1}$. Raw time series were extracted and averaged in each ROI within ventral default mode network (vDMN), dorsal default mode network (dDMN), anterior salience network (aSN), posterior salience network (pSN), and SMN (Shirer et al., 2012) (see Supplementary Table 1 for detailed information about the ROIs).

\section{Measures}

For the purpose of developing a predictive classification model, the authors used Regional Homogeneity (ReHo), Amplitude of Low Frequency Fluctuations (ALFF), Fractional Amplitude of Low Frequency Fluctuations (fALFF), and Functional Connectivity (FC). Each measure has its static and dynamic equivalent, and each measure was extracted for both atlases (see Figure 1 for study flowchart and Supplementary Materials for detailed description of the measures).

\section{Stationary Approach}

The mReHo, ALFF, and f/ALFF maps were segmented into 116 and 264 brain regions, and the values were extracted accordingly. Each participant got $116 \times 1$ (AAL atlas) and $264 \times 1$ (Power et al. atlas) matrices, consisting of a single value for each brain region among all 390 time points. Z-transformed correlation coefficients were obtained for each brain atlas, which resulted in $264 \times 264$ as well as $116 \times 116$ matrices for each participant. The lower half and diagonal values were removed and not used as features for ML algorithms, so that the total of 6,670 and 34,716 $z$-score values were used.

\section{Dynamic Approach}

The dynamic mALFF, mf/ALFF, and mReHo indexes were computed using the Temporal Dynamic Analysis module of Dpabi (Yan et al., 2017) using a sliding window approach with a hamming window shape.

According to previous work of Leonardi and Van De Ville (2015), in order to reduce the likelihood of spurious fluctuations in the dynamics of observed data, minimum window length should have at least $1 / f_{\min }$, where $f_{\min }$ is the minimum frequency of the time series; a similar approach was used among others in the work of Li et al. (2019), where the authors showed alterations in temporal dynamics of the brain associated with suicidal ideations in depression. In our case, $f_{\min }$ after band-pass filter equals $0.01 \mathrm{~Hz}(100 \mathrm{~s})$, and for this reason, window size was set to 50 TR (103 s) length and was shifted by 1 TR (2.06 s). The full time series was divided into 341 windows for each participant. As in case of stationary maps, dynamic maps were segmented using AAL and Power atlases. As a result, each participant gets a matrix of size $341 \times 116$ for AAL atlas and $341 \times 264$ for Power et al. atlas for dynamic ReHo (dReHo), dynamic ALFF (dALFF), as well as dynamic f/ALFF (df/ALFF). Each column represented a brain region and rows were populated with a single value for each window. Dynamic functional connectivity

\footnotetext{
${ }^{1}$ http://findlab.stanford.edu/functional_ROIs.html
} 


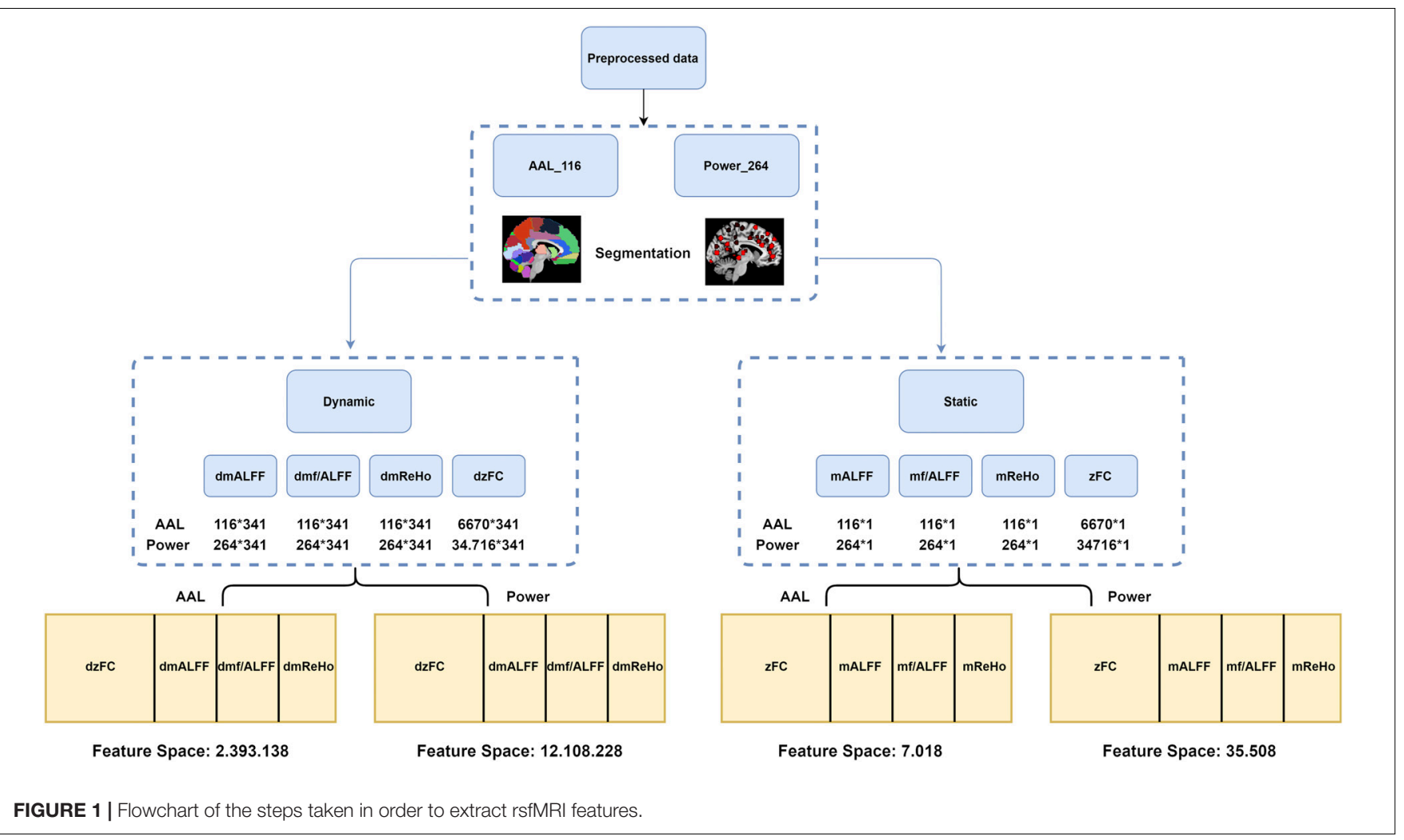

(dFC) was computed using the same window size, shift, and method via DynamicBC v.2.2 (Liao et al., 2014). After the calculations, data were represented by a $341 \times 116 \times 116$ matrix for AAL atlas and $341 \times 264 \times 264$ for Power atlas, where each of the 341 windows were "populated" with $z$-score Pearson correlation values. As in the case of stationary FC, upper half and diagonal values were removed from each of the 341 matrices so that 6670 and $34,716 z$-score values for each window were used, which gave a total of 2.274470 (aal) and 11.838156 (Power) $z$-score values used as an input for classification algorithms.

\section{Classification Models}

The authors used a selection of the most effective classification algorithms, each with a different level of complexity: LR (Cramer, 2002), LASSO (Tibshirani, 1996), SVM (Boser et al., 1992), RF (Ho, 1995), and GB (Friedman, 2001). See Supplementary Materials for a detailed description of the algorithms. Python, SciPy, NumPy, and scikit-learn (ver. 0.21) (Pedregosa et al., 2011) were used to compute the results. Standard scikit-learn model classes, score calculation routines, grid search, and dataset splitting functions were applied where possible.

The source code is available at https:/github.com/gmum/ schizo_fmri.

\section{Classification Framework}

The dataset was divided into train and test sets of approximately equal sizes (19 and 20 patients, respectively) with stratification. The training dataset was used to train a classifier pipeline consisting of optional data standardization and dimensionality reduction using Principal Component Analysis (PCA) steps and of the actual classifier. A grid search with fivefold cross-validation was performed to find the optimal hyperparameters. The entire hyperparameter grid search and training procedure is illustrated in Figure 2. It was run separately for every combination of the classifier type, input data type, and whether dimensionality reduction was performed.

For static data, the model simply accepts the entire data of selected measure types for the given patient. For dynamic data, the model is given only a single window and, thus, predicts a binary label for each window. An aggregation scheme is needed to make a final prediction for a patient. For this purpose, we applied a simple thresholding and counting scheme. Window results are first transformed to binary results using 0.5 as the threshold value. The final result was defined as the ratio of positive results to the total number of windows.

\section{Classification Performance}

Area Under Receiver Operating Curve (AUROC) was used as the score metric for grid search cross-validation procedure, and both AUROC and accuracy of the final classifier were evaluated on the test dataset.

In addition, the $p$-value of each AUROC score was calculated in order to determine the statistical significance of the obtained results. To do this, 1,000 permutations of the target labels were generated. Then, for each permutation, the classifier was retrained on the permuted labels, and its AUROC score was measured. The $p$-value was defined as the ratio of runs that 


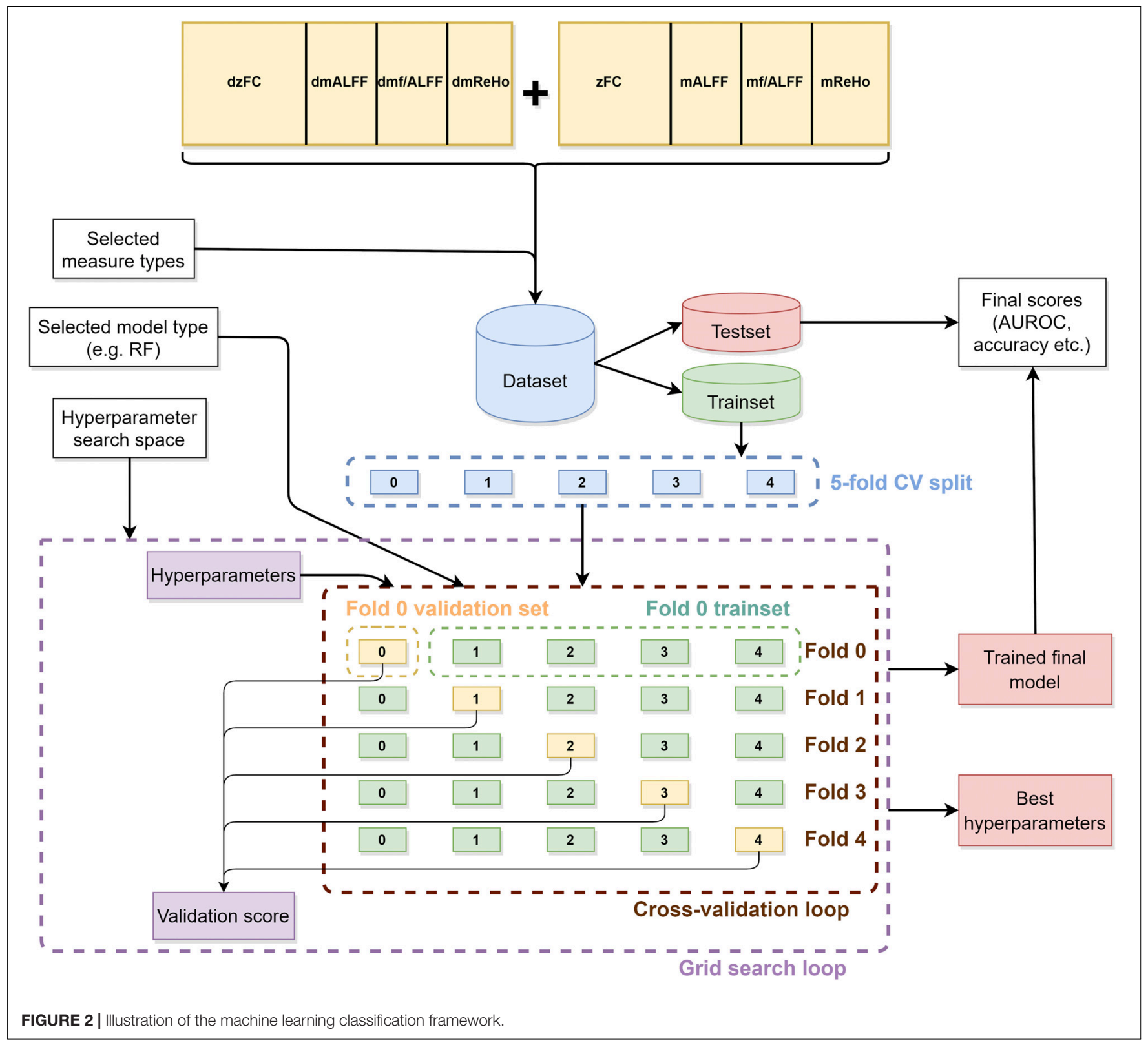

achieved a score greater than or equal to the score of the original classifier (Ojala and Garriga, 2010).

\section{Statistical Analysis}

Two-sample $t$-tests were used in order to seek for possible differences in suicidal risk (SBQ-R), mental pain (TPS), severity, and illness duration between the SR and NSR groups. One-way analysis of variance (ANOVA) was used to check for possible differences in age, mean FD, and education (in years) between SR, NSR, and HCs groups, and the Kruskal-Wallis $H$ test was used to compare gender differences.

One-way ANOVA was used to investigate possible differences in FC among three rsfMRI networks between SR, NSR, and HCs groups. The results were corrected with the Benjamini and Hochberg (1995) False Discovery Rate correction at $p<0.05$.
One-way ANCOVA with the Bonferroni post hoc test was used to investigate the differences in static ALFF, fALFF, ReHo between SR, NSR, and HCs. Age, gender, and mean FD were used as covariates. The same set of analyses was applied to compare the group-level temporal variability of ALFF, fALFF, ReHo, and FC. Temporal variability for ALFF, fALFF, and ReHo was expressed as a coefficient of variations (SD/mean) and, for FC, as a variance calculated across sliding-window dynamics, and then compared using one-way ANOVA with FDR correction. The statistical significance level for ALFF, fALFF, and ReHo analyses was set as $p_{F W E R}<0.05$ with 5,000 times permutation using Permutation Analysis of Linear Models (Winkler et al., 2016) as a part of DPABI. The cluster forming threshold was set to $z=2.3$, which is equal to $p<0.01$ and the cluster extent threshold at $k>25$ (Li et al., 2019). 


\section{RESULTS}

\section{Demographic and Clinical Characteristics}

The final analysis was conducted based on the data obtained from 59 participants, 39 of whom were schizophrenia patients. No differences in age [one-way ANOVA; $F_{(2,57)}=2.13 ; p=0.1282$ ], gender [Kruskal-Wallis; $H_{(2)}=2,468 ; p=0.291$ ], and head motion [one-way ANOVA; $F_{(2,57)}=0.66 ; p=0.5214$ ] were found among the three groups. SR and NSR groups were significantly different in SBQ-R score $(t=7.645 ; p<0.001)$ and illness duration $(t=1.69 ; p=0.01)$, but no differences were found in the case of TPS scores $(t=1.904 ; p=0.064)$ The range of SBQ-R score in the SR group was 8-17 points (Table 1).

\section{Differences in rsfMRI Measures}

No differences were found among the three groups in static ALFF, fALFF, and ReHo. No significant differences were found in the case of temporal variability of ALFF, fALFF, or ReHo either. Significant differences between SR, NSR, and HCs groups were found in both static functional connectivity and temporal variability of FC. One-way ANOVA showed that the three
TABLE 1 | Detailed participant demographic and clinical information.

\begin{tabular}{lccc}
\hline Demographics & HCs & SR & NSR \\
\hline Group size $(n)$ & 20 & 20 & 19 \\
FD (0-3) & $0.079 \pm 0.04$ & $0.09 \pm 0.05$ & $0.097 \pm 0.063$ \\
TPS (13-59) & $15.38 \pm 6.88$ & $33.65 \pm 10.24$ & $26.57 \pm 12.86$ \\
SBQ-R (3-17) & $4.42 \pm 2.11$ & $10.7 \pm 2.97$ & $5.10 \pm 1.32$ \\
Gender (female/male) & $10 / 10$ & $5 / 15$ & $9 / 10$ \\
Age (27-65) & $36.57 \pm 7.25$ & $42.6 \pm 9.4$ & $39.1 \pm 9.23$ \\
Handedness (right/left) & $20 / 0$ & $20 / 0$ & $19 / 0$ \\
Illness duration (years) (10-39) & - & $18 \pm 10.1$ & $10.89 \pm 5.93$ \\
\hline
\end{tabular}

SBQ-R, Suicide Behaviors Questionnaire-Revised; TPS, The Psychache Scale. Ranges of the variables are provided in the parentheses.

groups were different in FC among ventral DMN ( $F=19.02$; $p<0.001)$ and anterior SN $(F=6.85 ; p=0.001)$ (Figure 3$)$. Post hoc tests showed that the significant differences among ventral DMN network were present between SR and NSR groups $(p<0.001$; FDR corrected) and NSR and HCs groups $(p<0.01$; FDR corrected). In the case of anterior $\mathrm{SN}$, post hoc tests indicated differences between SR and NSR groups $(p=0.03$; FDR corrected). No differences were found among dorsal DMN,

\section{A}

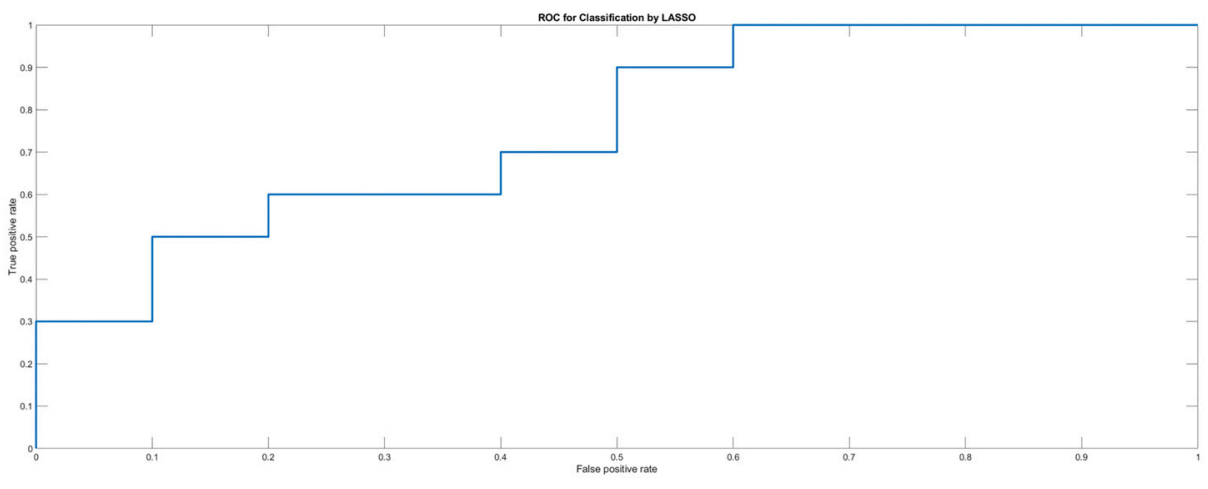

B

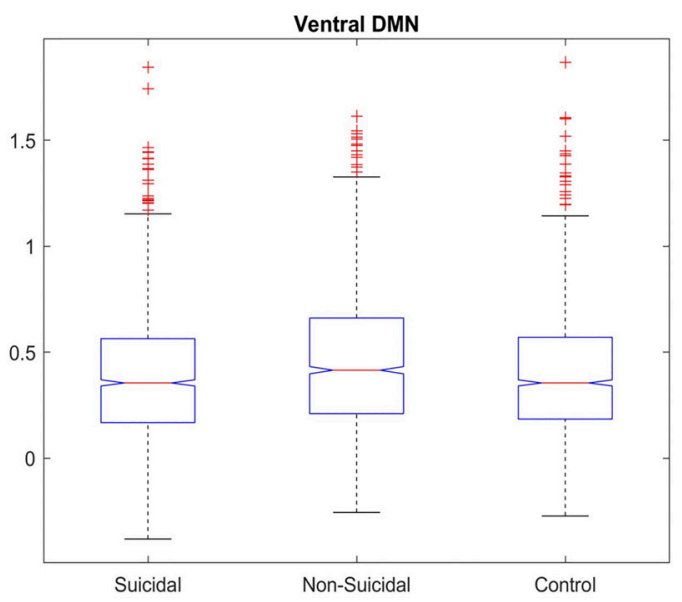

C

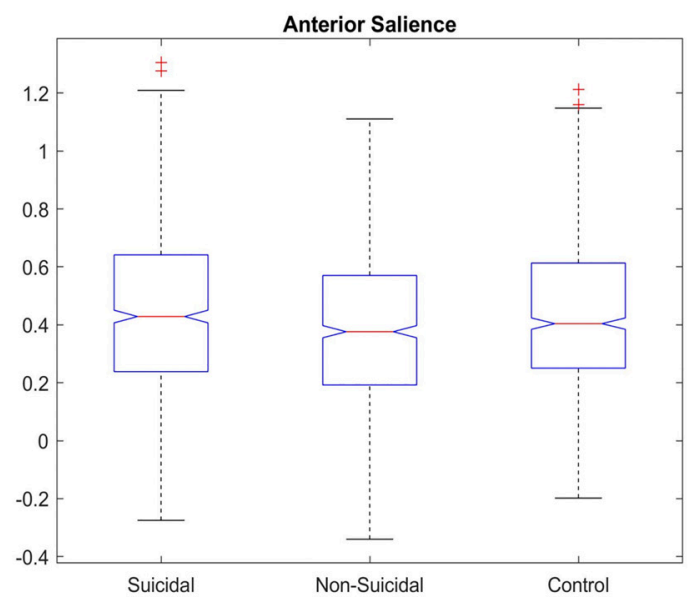

FIGURE 3 | (A) Receiver operating characteristic curve for LASSO classificator applied to Functional Connectivity with Power 264 atlas. (B) Box-and-whisker plot illustrating the differences between three groups in Functional Connectivity among ventral Default Mode Network. (C) Box-and-whisker plot illustrating the differences between three groups in Functional Connectivity among Anterior Salience Network. The outliers can be seen due to the high variance of dFC values. 
TABLE 2 | Dynamic functional connectivity and functional connectivity differences among NSR, SR, and HC groups.

\begin{tabular}{|c|c|c|c|}
\hline Region & $\boldsymbol{F}$ & $p$ & Pairwise comparison \\
\hline Dorsal DMN & 1.55 & 0.2133 & - \\
\hline Ventral DMN & 19.02 & $<0.001$ & $\begin{array}{l}\text { SR vs. NSR }(p<0.0001, M D=-0.0582) \\
\text { NSR vs. } \mathrm{HCs}(p<0.0001, \mathrm{MD}=0.0550)\end{array}$ \\
\hline Anterior SN & 6.85 & 0.0011 & $\begin{array}{l}\text { SR vs. NSR }(p=0.001, M D=0.0482) \\
\text { NSR vs. HCs }(p=0.029, M D=-0.0347)\end{array}$ \\
\hline Posterior SN & 1.23 & 0.2919 & - \\
\hline $\begin{array}{l}\text { Sensorimotor } \\
\text { Network }\end{array}$ & 1.66 & 0.1898 & - \\
\hline $\begin{array}{l}\text { Dynamic } \\
\text { variance Power } \\
\text { Atlas FC }\end{array}$ & 677.67 & $<0.001$ & $\begin{array}{l}\text { SR vs. NSR }(p<0.0001, M D=-0.00103) ; \\
\text { SR vs. HCs }(p<0.0001, M D=-0.00095) ; \\
\text { NSR vs. HCs }(p=0.0404, \\
M D=0.0000775)\end{array}$ \\
\hline $\begin{array}{l}\text { Dynamic } \\
\text { variance } A A L \\
\text { FC }\end{array}$ & 108.61 & $<0.001$ & $\begin{array}{l}\text { SR vs. NSR }(p<0.0001, M D=-0.00098) ; \\
\text { SR vs. HCs }(p<0.0001, M D=-0.00068) ; \\
\text { NSR vs. HCs }(p=0.00031 \\
M D=0.000303)\end{array}$ \\
\hline
\end{tabular}

Statistical differences were computed using one-way ANOVA with FDR correction.

posterior $\mathrm{SN}$, or SMN between the three groups. Temporal variability of FC, calculated at each voxel for the two atlases, showed that the three groups were significantly $(p<0.001)$ different in total FC variability measured using AAL, as well as Power atlases. Post hoc tests with FDR correction showed that in the case of both atlases, FC variability was significantly different between SR and NSR, between SR and HCs, and between NSR and HCs groups (Table 2 and Supplementary Figures 1, 2).

\section{Classification Results}

The 10 most important features of amplitude of low-frequency fluctuations (ALFF) for LR as well as of Functional Connectivity for LASSO classifiers are presented in Table 3. The accuracies and AUROCs of five ML algorithms for each static and dynamic rsfMRI measures, divided into two atlases, are listed in Table 4.

Three variants of ML algorithm and rsfMRI measures turned out to be significant at $p<0.05$. (1) The LASSO applied to static functional connectivity with Power atlas reached an accuracy of $70 \%$ and an AUROC of 0.76. (2) The LR algorithm applied to dynamic ALFF with AAL atlas reached an accuracy of $65 \%$ and an AUROC of 0.75. (3) The GB algorithm applied to static fALFF with AAL atlas reached an accuracy of $65 \%$ and an AUROC of 0.74. It can be seen that AUROCs of the majority of variants were at chance level, even when accuracies were above $50 \%$. In short, the obtained results suggest that the combination of LASSO algorithm and static functional connectivity calculated on 264 ROIs provide superior accuracy/AUROC of classification between SR patients and non-SR patients and allow correct classification of 14 out of 20 SR patients.

\section{DISCUSSION}

In the present article, a successful discrimination between schizophrenia patients with and without a suicide risk using ML algorithms and rsfMRI data was demonstrated.
TABLE 3 | The 10 most important features for Logistic Regression and LASSO classifiers.

\begin{tabular}{lcc}
\hline Ensemble Method & Logistic Regression & \\
\cline { 1 - 2 } Feature & ROI labels & $\begin{array}{c}\text { Coordinates } \\
\text { (MNI) }\end{array}$ \\
\hline 1 & Angular_L & $-39,-74,43$ \\
2 & Precuneus_L & $-11,-56,15$ \\
3 & Cingulum_Ant_L & $-2,41,16$ \\
4 & Temporal_Sup_L & $-60,-25,13$ \\
5 & Postcentral_R & $65,-7,24$ \\
6 & Precentral_R & $20,-29,60$ \\
7 & Postcentral_R & $50,-20,42$ \\
8 & Postcentral_L & $-53,-10,24$ \\
9 & Rolandic_Oper_R & $43,-23,20$ \\
10 & Frontal_Sup_L & $-20,45,39$
\end{tabular}

\begin{tabular}{|c|c|}
\hline Ensemble Method & LASSO \\
\hline Feature & ROI-ROI labels \\
\hline 1 & Supramarginal_R-Precentral_R \\
\hline 2 & Postcentral_R-Precuneus_R \\
\hline 3 & Angular_R-Precentral_R \\
\hline 4 & Frontal_Sup_Medial_R-Parietal_Sup_R \\
\hline 5 & Paracentral_Lobule_L-Postcentral_R \\
\hline 6 & Frontal_Sup_Medial_R-Frontal_Mid_R \\
\hline 7 & Precentral_R-Frontal_Sup_Medial_R \\
\hline 8 & Frontal_Inf_Tri_L-Occipital_Mid_L \\
\hline 9 & Precentral_R-Frontal_Sup_Medial_R \\
\hline 10 & Frontal_Sup_Medial_L-Precentral_R \\
\hline
\end{tabular}

Data are presented for (a) Logistic Regression applied to dynamic amplitude of low-frequency fluctuations with AAL atlas and (b) LASSO applied to static ROI-ROI Functional Connectivity with Power 264 atlas.

Although previous studies developed the rsfMRI-based ML classification models capable of distinguishing suicidal patients with different diagnoses, such as anxiety disorder, depression, or borderline personality disorder (Gosnell et al., 2019; Wang et al., 2020), to the best of the authors' knowledge, none of the previous studies were focused on schizophrenia. Ipso facto, the presented study is the first attempt to find rsfMRI features that allow detecting the risk of suicide in schizophrenia with the use of the ML algorithms. Moreover, this is the first work using rsfMRI to explain the differences in brain activity, which might be associated with suicide risk in schizophrenia patients. Above that, unlike most of ML-fMRI studies focused on classifying suicidal participants, the authors used five various ML classifiers. The results of conventional analyses showed that patients with and without suicidal risk, as well as the healthy controls, demonstrated different patterns of temporal variability of $\mathrm{dFC}$ and $\mathrm{FC}$, with the latter being an important feature for ML classification. Furthermore, ALFF and fALFF measures also contributed to ML-based classification, but no significant differences in the above measures were found in the conventional group analyses.

Du et al. (2015) and also Gosnell et al. (2019) results are partly congruent with the ones obtained from this study, 
TABLE 4 | The accuracies and AUROCs of five ML algorithms for each static and dynamic rsfMRI measures, divided into two atlases.

\begin{tabular}{|c|c|c|c|c|c|c|c|c|c|c|}
\hline \multirow[b]{2}{*}{ AAL 116 Static } & \multicolumn{2}{|c|}{ ALFF } & \multicolumn{2}{|c|}{ fALFF } & \multicolumn{2}{|r|}{ FC } & \multicolumn{2}{|c|}{ REHO } & \multicolumn{2}{|c|}{ Concatenated } \\
\hline & With PCA & Without PCA & With PCA & Without PCA & With PCA & Without PCA & With PCA & Without PCA & With PCA & Without PCA \\
\hline GB & $55 \% / 0.6$ & $55 \% / 0.62$ & $65 \% / 0.74^{\star}$ & $40 \% / 0.25$ & $65 \% / 0.65$ & $55 \% / 0.47$ & $45 \% / 0.46$ & $45 \% / 0.43$ & $55 \% / 0.62$ & $55 \% / 0.49$ \\
\hline LASSO & $55 \% / 0.41$ & $50 \% / 0.41$ & $55 \% / 0.54$ & $65 \% / 0.61$ & $50 \% / 0.58$ & $50 \% / 0.65$ & $65 \% / 0.54$ & $50 \% / 0.6$ & $50 \% / 0.27$ & $45 \% / 0.55$ \\
\hline LR & $60 \% / 0.55$ & $45 \% / 0.5$ & $35 \% / 0.29$ & $35 \% / 0.34$ & $35 \% / 0.34$ & $35 \% / 0.32$ & $35 \% / 0.36$ & $35 \% / 0.32$ & $45 \% / 0.43$ & $35 \% / 0.29$ \\
\hline $\mathrm{RF}$ & $45 \% / 0.57$ & $50 \% / 0.55$ & $65 \% / 0.66$ & $30 \% / 0.34$ & $50 \% / 0.43$ & $60 \% / 0.44$ & $30 \% / 0.32$ & $25 \% / 0.24$ & $55 \% / 0.67$ & $50 \% / 0.36$ \\
\hline SVM & $50 \% / 0.46$ & $50 \% / 0.42$ & $50 \% / 0.55$ & $40 \% / 0.29$ & $50 \% / 0.14$ & $40 \% / 0.37$ & $50 \% / 0.38$ & $30 \% / 0.34$ & $45 \% / 0.38$ & $60 \% / 0.48$ \\
\hline \multicolumn{11}{|c|}{ AAL 116 Dynamic } \\
\hline $\mathrm{GB}$ & $45 \% / 0.47$ & $60 \% / 0.5$ & $40 \% / 0.42$ & $50 \% / 0.5$ & - & - & $60 \% / 0.5$ & $40 \% / 0.29$ & - & - \\
\hline LASSO & $40 \% / 0.45$ & $50 \% / 0.39$ & $60 \% / 0.57$ & $50 \% / 0.45$ & - & - & $40 \% / 0.43$ & $55 \% / 0.49$ & - & - \\
\hline LR & $50 \% / 0.65$ & $65 \% / 0.75^{\star}$ & $60 \% / 0.56$ & $35 \% / 0.39$ & $35 \% / 0.27$ & - & $50 \% / 0.51$ & $35 \% / 0.41$ & $45 \% / 0.44$ & - \\
\hline $\mathrm{RF}$ & $45 \% / 0.49$ & $50 \% / 0.57$ & $35 \% / 0.48$ & $45 \% / 0.47$ & $50 \% / 0.43$ & - & $35 \% / 0.38$ & $40 \% / 0.41$ & $70 \% / 0.6$ & - \\
\hline SVM & $45 \% / 0.46$ & $45 \% / 0.42$ & $40 \% / 0.42$ & $40 \% / 0.41$ & - & - & $45 \% / 0.32$ & $55 \% / 0.39$ & - & - \\
\hline \multicolumn{11}{|l|}{ Power 264 Static } \\
\hline $\mathrm{GB}$ & $60 \% / 0.55$ & $40 \% / 0.5$ & $40 \% / 0.32$ & $55 \% / 0.56$ & $45 \% / 0.67$ & $50 \% / 0.5$ & $40 \% / 0.4$ & $30 \% / 0.33$ & $50 \% / 0.62$ & $50 \% / 0.5$ \\
\hline LASSO & $45 \% / 0.42$ & $25 \% / 0.42$ & $70 \% / 0.65$ & $55 \% / 0.31$ & $50 \% / 0.41$ & $70 \% / 0.76$ & $35 \% / 0.34$ & $35 \% / 0.36$ & $45 \% / 0.51$ & $50 \% / 0.41$ \\
\hline LR & $55 \% / 0.61$ & $60 \% / 0.48$ & $45 \% / 0.51$ & $40 \% / 0.44$ & $45 \% / 0.47$ & $45 \% / 0.43$ & $50 \% / 0.51$ & $40 \% / 0.45$ & $45 \% / 0.48$ & $45 \% / 0.4$ \\
\hline $\mathrm{RF}$ & $60 \% / 0.65$ & $40 \% / 0.41$ & $45 \% / 0.47$ & $55 \% / 0.46$ & $60 \% / 0.69$ & $55 \% / 0.38$ & $55 \% / 0.68$ & $70 \% / 0.57$ & $55 \% / 0.48$ & $50 \% / 0.49$ \\
\hline SVM & $50 \% / 0.34$ & $50 \% / 0.39$ & $50 \% / 0.45$ & $50 \% / 0.33$ & $50 \% / 0.68$ & $50 \% / 0.46$ & $45 \% / 0.24$ & $40 \% / 0.43$ & $50 \% / 0.69$ & $50 \% / 0.46$ \\
\hline \multicolumn{11}{|c|}{ Power 264 Dynamic } \\
\hline $\mathrm{GB}$ & $55 \% / 0.58$ & $45 \% / 0.6$ & $50 \% / 0.5$ & $60 \% / 0.57$ & - & - & $55 \% / 0.6$ & $55 \% / 0.64$ & - & - \\
\hline LASSO & $60 \% / 0.5$ & $55 \% / 0.65$ & $45 \% / 0.62$ & $45 \% / 0.38$ & - & - & $45 \% / 0.58$ & $45 \% / 0.5$ & - & - \\
\hline LR & $60 \% / 0.57$ & $70 \% / 0.66$ & $55 \% / 0.51$ & $50 \% / 0.44$ & $50 \% / 0.46$ & - & $50 \% / 0.47$ & $50 \% / 0.5$ & $50 \% / 0.48$ & - \\
\hline $\mathrm{RF}$ & $45 \% / 0.44$ & $55 \% / 0.53$ & $50 \% / 0.34$ & $50 \% / 0.48$ & $50 \% / 0.41$ & - & $40 \% / 0.61$ & $60 \% / 0.55$ & $50 \% / 0.49$ & - \\
\hline SVM & $45 \% / 0.46$ & $45 \% / 0.6$ & $40 \% / 0.47$ & $55 \% / 0.42$ & - & - & $50 \% / 0.55$ & $50 \% / 0.51$ & - & - \\
\hline
\end{tabular}

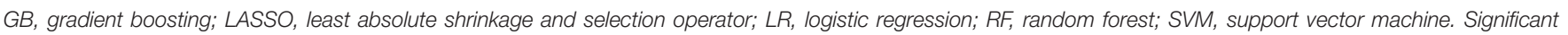

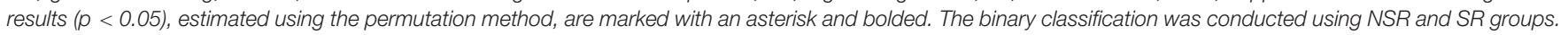

indicating frontal and temporal brain region abnormalities to be the most useful in ML classification of suicidal risk, suggesting activity of specific brain structures to be characteristic for suicide risk among all psychiatric patients. Cao et al. (2015), in turn, demonstrated altered ReHo in suicide attempters without psychiatric diagnosis in left precuneus, which also remains consistent with the region allowing discriminating between SR and NSR patients reported in our study. In addition, regions that turned out to be discriminative in ML-based classification are also congruent with the studies using cognitive control tasks, revealing the association between decreased activity of the frontal cortex and suicide risk in schizophrenia (Zhang et al., 2013; Potvin et al., 2018). Our results are also consistent with the studies showing significance of ACC, angular gyrus, as well as both precentral and postcentral gyrus in understanding the suicidal behavior (Reisch et al., 2010; Fan et al., 2013; Tsujii et al., 2017; Harms et al., 2019).

Numerous fMRI studies indicate the association of suiciderelated behaviors and prefrontal cortex alterations due to its role in decision-making as well as action planning (Potvin et al., 2018; Brown et al., 2020). Other studies suggest decreased connectivity between ACC and PFC to be related to suicidal behavior (Minzenberg et al., 2015; Chase et al., 2017). PFC and ACC are considered responsible for anticipating the consequence of actions, inhibition of inappropriate behavior, and impulsiveness (Zhou et al., 2016; Brown et al., 2019), which are indirectly related to suicidal behavior (Wang et al., 2017; Koval and Baumann, 2019). Alterations in ACC and PFC have also been found in patients with schizophrenia (Cordes et al., 2015; Fryer et al., 2019) while their impulsivity has been reported as correlated with increased suicide risk (Iancu et al., 2010). Notably, the above regions are included in DMN as well as SN, which have already been established as disrupted in schizophrenia (Garrity et al., 2007; White et al., 2010; Palaniyappan et al., 2012). Weaker functional connectivity within DMN is reported to be associated with the difficulties in abstract thinking, planning the future, as well as analyzing social behaviors (Andrews-Hanna, 2012), while decreased functional connectivity within $\mathrm{SN}$ has been reported as linked to higher trait anxiety and decreased cognitive regulation (Geng et al., 2016). The above symptoms might additionally elevate suicidal risk.

Importantly, our results from conventional static FC analyses confirm the aforementioned results, revealing functional connectivity differences in ventral DMN and anterior SN between SR and NSR patients. The above dissimilarity was also apparent between NSR patients and HCs. Moreover, The LASSO algorithm, applied on static functional connectivity data, allowed the discrimination between SR and NSR patients, supporting our hypothesis. Furthermore, all the three groups varied from each other in the case of temporal variability of dFC with the use of both AAL and Power atlases. 
According to another hypothesis, all five ML algorithms presented different classification performance. The LASSO, applied on static functional connectivity without PCA on Power atlas, achieved $70 \%$ accuracy and an AUROC of 0.76, proving to be the best ML classifier used in the study. As a result, LASSO allowed the correct classification of 14 out of 20 suicidal patients. The possible reason why the LASSO outperforms the other classifiers is the type of its regularization loss term. The processed dataset has high dimensionality but very few samples, which could possibly make the standard classifiers heavily overfit to the train set and therefore perform poorly on the test set. As a result, a regularization method needs to be used in order to reduce overfitting and increase generalization. The L1 cost used in LASSO has a property of much stronger parameters' shrinkage due to its diagonal regularization contour, leading to a more sparse model.

Noteworthy, raw performance of the classifiers differed depending on the selected parcellation scheme. However, contrary to our assumptions, the dynamic measures did not improve the prediction ability of ML classifiers compared to static measures. As far as the authors are aware, none of the previous studies used ML-based classifiers to discriminate between SR and NSR schizophrenia patients. Further studies should consider to enlarge sample size in order to demonstrate the replicability of our study.

\section{LIMITATIONS}

The conducted study has some limitations. Firstly, EPI sequence was introduced to participants after the structural scans (T1MPRAGE) and not before them, which could influence the results. Secondly, results may possibly depend on the size of the smoothing kernel. Another noteworthy limitation of this study is the restricted sample size; thus, the presented results should be interpreted with caution. Further studies should consider extending the sample size by adding the control group to the input training data. Moreover, the specific window size as well as arbitrarily chosen atlases could influence the results. What is more, the high number of features may cause high susceptibility to any noise signal; therefore, distinctive features could be possibly different in another sample size, i.e., patients. Based on the literature, we have also decided to include the global signal, which is not always considered to be beneficial. Additionally, further studies should incorporate a semi-supervised approach with a pre-training phase using the data of $\mathrm{HC}$.

\section{REFERENCES}

Andrews-Hanna, J. R. (2012). The brain's default network and its adaptive role in internal mentation. Neuroscientist 18, 251-270. doi: 10.1177/ 1073858411403316

Behzadi, Y., Restom, K., Liau, J., and Liu, T. T. (2007). A component based noise correction method (CompCor) for BOLD and perfusion based fMRI. Neuroimage 37, 90-101. doi: 10.1016/j.neuroimage.2007. 04.042

\section{DATA AVAILABILITY STATEMENT}

The raw data supporting the conclusions of this article will be made available by the authors, without undue reservation, to any qualified researcher.

\section{ETHICS STATEMENT}

The studies involving human participants were reviewed and approved by Jagiellonian University Bioethics Committee. The patients/participants provided their written informed consent to participate in this study.

\section{AUTHOR CONTRIBUTIONS}

BB: conceptualization, methodology, validation, formal analysis, investigation, writing - original draft, writing - review and editing, visualization, and project administration. AMS: formal analysis, investigation, writing - original draft, writing - review and editing, and visualization. IP: methodology, software, validation, writing - review and editing, and supervision. BW: software, formal analysis, writing - original draft, and visualization. DM: resources and writing - original draft. AC: investigation, resources, and funding acquisition. MF: writing - review and editing. MS: resources. DD: resources. TM: resources, writing - review and editing, supervision, and funding acquisition. All authors contributed to the article and approved the submitted version.

\section{FUNDING}

This work and doctoral dissertation preparation were supported by a doctoral scholarship from the Polish National Science Centre (NCN) (ETIUDA, ID: 2019/32/T/HS6/00339) for BB. The work was also supported by the Foundation for Polish Science (FNP) project (Bio-inspired Artificial Neural Networks ID: POIR.04.04.00-00-14DE/18-00) for BB, IP, AMS, MF, and TM.

\section{SUPPLEMENTARY MATERIAL}

The Supplementary Material for this article can be found online at: https://www.frontiersin.org/articles/10.3389/fnins. 2020.605697/full\#supplementary-material

Benjamini, Y., and Hochberg, Y. (1995). Controlling the false discovery rate: a practical and powerful approach to multiple testing. J. R. Statist. Soc. Ser. B 57, 289-300. doi: 10.1111/j.2517-6161.1995.tb02031.x

Besteher, B., Wagner, G., Koch, K., Schachtzabel, C., Reichenbach, J. R., Schlösser, R., et al. (2016). Pronounced prefronto-temporal cortical thinning in schizophrenia: neuroanatomical correlate of suicidal behavior? Schizophre. Res. 176, 151-157. doi: 10.1016/j.schres.2016.08.010

Bohaterewicz, B., Jabłońska, A., and Szulc, A. (2018). Suicidal thoughts and behaviors and their comorbidity with psychopathological symptoms 
among patients with schizophrenia hospitalized at long term ward. Studia Psychol. 56, 21-31.

Boser, B. E., Guyon, I. M., and Vapnik, V. N. (1992). "A training algorithm for optimal margin classifiers," in Proceedings of the Fifth Annual Workshop on Computational Learning Theory, New York, NY.

Brett, M., Anton, J. L., Valabregue, R., and Poline, J. B. (2002). Region of interest analysis using the MarsBar toolbox for SPM 99. Neuroimage 16, S497.

Brown, V., Wilson, J., Szanto, K., and Dombrovski, A. (2019). F77. Ventromedial prefrontal functional connectivity during value-based decision making in Impulsive vs. Non-Impulsive older suicide attempters. Biol. Psychiatry 85, S242-S243.

Brown, V. M., Wilson, J., Hallquist, M. N., Szanto, K., and Dombrovski, A. Y. (2020). Ventromedial prefrontal value signals and functional connectivity during decision-making in suicidal behavior and impulsivity. Neuropsychopharmacology 45, 1034-1041. doi: 10.1038/s41386-020-0632-0

Buchanan, R. W., and Heinrichs, D. W. (1989). The Neurological Evaluation Scale (NES): a structured instrument for the assessment of neurological signs in schizophrenia. Psychiatry Res. 27, 335-350. doi: 10.1016/0165-1781(89) 90148-0

Cao, J., Chen, J. M., Kuang, L., Ai, M., Fang, W. D., Gan, Y., et al. (2015). Abnormal regional homogeneity in young adult suicide attempters with no diagnosable psychiatric disorder: a resting state functional magnetic imaging study. Psychiatry Res. Neuroimaging 231, 95-102. doi: 10.1016/j.pscychresns. 2014.10.011

Cassidy, R. M., Yang, F., Kapczinski, F., and Passos, I. C. (2018). Risk factors for suicidality in patients with schizophrenia: a systematic review, meta-analysis, and meta-regression of 96 studies. Schizophr. Bull. 44, 787-797. doi: 10.1093/ schbul/sbx131

Chase, H. W., Segreti, A. M., Keller, T. A., Cherkassky, V. L., Just, M. A., Pan, L. A., et al. (2017). Alterations of functional connectivity and intrinsic activity within the cingulate cortex of suicidal ideators. J. Affect. Disord. 212, 78-85. doi: 10.1016/j.jad.2017.01.013

Chodkiewicz, J. (2013). Ból psychiczny—konceptualizacja i narzędzia pomiaru. Psychiatria 10, 109-115.

Chodkiewicz, J., and Gruszczyńska, E. (2020). Polska adaptacja zrewidowanego kwestionariusza zachowań samobójczych A. Osmana i współpracowników. Psychiatr. Pol. 54, 101-111.

Cordes, J. S., Mathiak, K. A., Dyck, M., Alawi, E. M., Gaber, T. J., Zepf, F., et al. (2015). Cognitive and neural strategies during control of the anterior cingulate cortex by fMRI neurofeedback in patients with schizophrenia. Front. Behav. Neurosci. 9:169. doi: 10.3389/fnbeh.2015.00169

Cramer, J. S. (2002). The Origins of Logistic Regression. Tinbergen Institute Working Paper No. 2002-119/4. doi: 10.2139/ssrn.360300

Du, Y., Pearlson, G. D., Liu, J., Sui, J., Yu, Q., He, H., et al. (2015). A group ICA based framework for evaluating resting fMRI markers when disease categories are unclear: application to schizophrenia, bipolar, and schizoaffective disorders. Neuroimage 122, 272-280. doi: 10.1016/j.neuroimage.2015.07.054

Ducasse, D., Holden, R. R., Boyer, L., Artero, S., Calati, R., Guillaume, S., et al. (2018). Psychological pain in suicidality: a meta-analysis. J. Clin. Psychiatry 79:16r10732.

Fan, T., Wu, X., Yao, L., and Dong, J. (2013). Abnormal baseline brain activity in suicidal and non-suicidal patients with major depressive disorder. Neurosci. Lett. 534, 35-40. doi: 10.1016/j.neulet.2012.11.032

Friedman, J. H. (2001). Greedy function approximation: a gradient boosting machine. Ann. Statist. 29, 1189-1232.

Friston, K. J. (1994). Statistical Parametric Mapping.

Friston, K. J., Holmes, A. P., Worsley, K. J., Poline, J.-P., Frith, C. D., and Frackowiak, R. S. J. (1994). Statistical parametric maps in functional imaging: a general linear approach. Hum. Brain Mapp. 2, 189-210. doi: 10.1002/hbm. 460020402

Fryer, S. L., Roach, B. J., Ford, J. M., Donaldson, K. R., Calhoun, V. D., Pearlson, G. D., et al. (2019). Should i stay or should i go? FMRI study of response inhibition in early illness schizophrenia and risk for psychosis. Schizophr. Bull. 45, 158-168. doi: 10.1093/schbul/sbx198

Garrity, A. G., Pearlson, G. D., McKiernan, K., Lloyd, D., Kiehl, K. A., and Calhoun, V. D. (2007). Aberrant "default mode" functional connectivity in schizophrenia. Am. J. Psychiatry 164, 450-457. doi: 10.1176/ajp.2007.164.3.450
Geng, H., Li, X., Chen, J., Li, X., and Gu, R. (2016). Decreased intra-and inter-salience network functional connectivity is related to trait anxiety in adolescents. Front. Behav. Neurosci. 9:350. doi: 10.3389/fnbeh.2015.00350

Glover, G. H. (2011). Overview of functional magnetic resonance imaging. Neurosurg. Clin. 22, 133-139.

Gosnell, S. N., Fowler, J. C., and Salas, R. (2019). Classifying suicidal behavior with resting-state functional connectivity and structural neuroimaging. Acta Psychiatr. Scand. 140, 20-29. doi: 10.1111/acps.13029

Harms, M. B., Casement, M. D., Teoh, J. Y., Ruiz, S., Scott, H., Wedan, R., et al. (2019). Adolescent suicide attempts and ideation are linked to brain function during peer interactions. Psychiatry Res. Neuroimaging 289, 1-9. doi: 10.1016/ j.pscychresns.2019.05.001

Ho, T. K. (1995). "Random decision forests," in Proceedings of 3rd International Conference on Document Analysis and Recognition, Montreal, QC.

Holden, R. R., Mehta, K., Cunningham, E. J., and McLeod, L. D. (2001). Development and preliminary validation of a scale of psychache. Can. J. Behav. Sci. 33:224. doi: $10.1037 / \mathrm{h} 0087144$

Iancu, I., Bodner, E., Roitman, S., Sapir, A. P., Poreh, A., and Kotler, M. (2010). Impulsivity, aggression and suicide risk among male schizophrenia patients. Psychopathology 43, 223-229. doi: 10.1159/000313520

Just, M. A., Pan, L., Cherkassky, V. L., McMakin, D. L., Cha, C., Nock, M. K., et al. (2017). Machine learning of neural representations of suicide and emotion concepts identifies suicidal youth. Natu. Hum. Behav. 1, 911-919. doi: 10.1038/ s41562-017-0234-y

Koval, P., and Baumann, R. (2019). The Impact of Impulsivity on Suicide and Homicide Rates. Working Papers 1907. Worcester, MA: College of the Holy Cross, Department of Economics.

Leonardi, N., and Van De Ville, D. (2015). On spurious and real fluctuations of dynamic functional connectivity during rest. Neuroimage 104, 430-436. doi: 10.1016/j.neuroimage.2014.09.007

Li, J., Duan, X., Cui, Q., Chen, H., and Liao, W. (2019). More than just statics: temporal dynamics of intrinsic brain activity predicts the suicidal ideation in depressed patients. Psychol. Med. 49, 852-860. doi: 10.1017/ s0033291718001502

Liao, W., Wu, G. R., Xu, Q., Ji, G. J., Zhang, Z., Zang, Y. F., et al. (2014). DynamicBC: a MATLAB toolbox for dynamic brain connectome analysis. Brain Connect. 4, 780-790. doi: 10.1089/brain.2014.0253

Liu, T. T., Nalci, A., and Falahpour, M. (2017). The global signal in fMRI: nuisance or Information? Neuroimage 150, 213-229. doi: 10.1016/j.neuroimage.2017.02. 036

Minzenberg, M. J., Lesh, T. A., Niendam, T. A., Yoon, J. H., Cheng, Y., Rhoades, R. N., et al. (2015). Control-related frontal-striatal function is associated with past suicidal ideation and behavior in patients with recent-onset psychotic major mood disorders. J. Affect. Disord. 188, 202-209. doi: 10.1016/j.jad.2015. 08.049

Minzenberg, M. J., Lesh, T. A., Niendam, T. A., Yoon, J. H., Rhoades, R. N., and Carter, C. S. (2014). Frontal cortex control dysfunction related to longterm suicide risk in recent-onset schizophrenia. Schizophr. Res. 157, 19-25. doi: 10.1016/j.schres.2014.05.039

Moghimi, P., Lim, K. O., and Netoff, T. I. (2018). Data driven classification using fMRI Network measures: application to schizophrenia. Front. Neuroinform. 12:71. doi: 10.3389/fninf.2018.00071

Ojala, M., and Garriga, G. C. (2010). Permutation tests for studying classifier performance. J. Mach. Learn. Res. 11, 1833-1863.

Osman, A., Bagge, C. L., Gutierrez, P. M., Konick, L. C., Kopper, B. A., and Barrios, F. X. (2001). The suicidal behaviors questionnaire-revised (SBQ-R): validation with clinical and nonclinical samples. Assessment 8, 443-454. doi: 10.1177/107319110100800409

Palaniyappan, L., White, T. P., and Liddle, P. F. (2012). The concept of salience network dysfunction in schizophrenia: from neuroimaging observations to therapeutic opportunities. Curr. Top. Med. Chem. 12, 2324-2338. doi: 10.2174/ 156802612805289881

Pedregosa, F., Varoquaux, G., Gramfort, A., Michel, V., Thirion, B., Grise, O., et al. (2011). Scikit-learn: machine learning in python. J. Mach. Learn. Res. 12, 2825-2830.

Pereira, F., Mitchell, T., and Botvinic, M. (2009). Machine learning classifiers and fMRI: a tutorial overview. Neuroimage 45, S199-S209. 
Potvin, S., Tikàsz, A., Richard-Devantoy, S., Lungu, O., and Dumais, A. (2018). History of suicide attempt is associated with reduced medial prefrontal cortex activity during emotional decision-making among men with schizophrenia: an exploratory fMRI study. Schizophr. Res. Treat. 2018:9898654.

Power, J. D., Cohen, A. L., Nelson, S. M., Wig, G. S., Barnes, K. A., Church, J. A., et al. (2011). Functional network organization of the human brain. Neuron 72 , 665-678.

Reisch, T., Seifritz, E., Esposito, F., Wiest, R., Valach, L., and Michel, K. (2010). An fMRI study on mental pain and suicidal behavior. J. Affect. Disord. 126, 321-325. doi: 10.1016/j.jad.2010.03.005

Shirer, W. R., Ryali, S., Rykhlevskaia, E., Menon, V., and Greicius, M. D. (2012). Decoding subject-driven cognitive states with whole-brain connectivity patterns. Cereb. Cortex 22, 158-165. doi: 10.1093/cercor/bhr099

Smitha, K. A., Akhil, R. K., Arun, K. M., Rajesh, P. G., Thomas, B., Kapilamoorthy, T. R., et al. (2017). Resting state fMRI: A review on methods in resting state connectivity analysis and resting state networks. Neuroradiol. J. 30, 305-317. doi: $10.1177 / 1971400917697342$

Tibshirani, R. (1996). Regression shrinkage and selection via the lasso. J. R. Statist. Soc. Ser. B 58, 267-288. doi: 10.1111/j.2517-6161.1996.tb02080.x

Tsujii, N., Mikawa, W., Tsujimoto, E., Adachi, T., Niwa, A., Ono, H., et al. (2017). Reduced left precentral regional responses in patients with major depressive disorder and history of suicide attempts. PLoS One 12:e0175249. doi: 10.1371/ journal.pone.0175249

Tzourio-Mazoyer, N., Landeau, B., Papathanassiou, D., Crivello, F., Etard, O., Delcroix, N., et al. (2002). Automated anatomical labeling of activations in SPM using a macroscopic anatomical parcellation of the MNI MRI single-subject brain. Neuroimage 15, 273-289. doi: 10.1006/nimg.2001. 0978

Wang, Y., Sun, K., Liu, Z., Chen, G., Jia, Y., Zhong, S., et al. (2020). Classification of unmedicated bipolar disorder using whole-brain functional activity and connectivity: a radiomics analysis. Cereb. Cortex 30, 1117-1128. doi: 10.1093/ cercor/bhz152

Wang, Y. G., Chen, S., Xu, Z. M., Shen, Z. H., Wang, Y. Q., He, X. Y., et al. (2017). Family history of suicide and high motor impulsivity distinguish suicide attempters from suicide ideators among college students. J. Psychiatr. Res. 90, 21-25. doi: 10.1016/j.jpsychires.2017.02.006

White, T. P., Joseph, V., Francis, S. T., and Liddle, P. F. (2010). Aberrant salience network (bilateral insula and anterior cingulate cortex) connectivity during information processing in schizophrenia. Schizophr. Res. 123, 105-115. doi: 10.1016/j.schres.2010.07.020

Wildgust, H. J., Hodgson, R., and Beary, M. (2010). The paradox of premature mortality in schizophrenia: new research questions. J. Psychopharmacol. 4(Suppl.), 9-15. doi: 10.1177/1359786810382149

Winkler, A. M., Ridgway, G. R., Douaud, G., Nichols, T. E., and Smith, S. M. (2016). Faster permutation inference in brain imaging. Neuroimage 141, 502-516. doi: 10.1016/j.neuroimage.2016.05.068

Yan, C. G., Wang, X. D., Zuo, X. N., and Zang, Y. F. (2016). DPABI: data processing $\&$ analysis for (resting-state) brain imaging. Neuroinformatics 14, 339-351. doi: 10.1007/s12021-016-9299-4

Yan, C. G., Yang, Z., Colcombe, S. J., Zuo, X. N., and Milham, M. P. (2017). Concordance among indices of intrinsic brain function: insights from interindividual variation and temporal dynamics. Sci. Bull. 62, 1572-1584. doi: 10.1016/j.scib.2017.09.015

Zhang, H., Wei, X., Tao, H., Mwansisya, T. E., Pu, W., He, Z., et al. (2013). Opposite effective connectivity in the posterior cingulate and medial prefrontal cortex between first-episode schizophrenic patients with suicide risk and healthy controls. PLoS One 8:e63477. doi: 10.1371/journal.pone.0063477

Zhang, R., Wei, S., Chang, M., Jiang, X., Tang, Y., and Wang, F. (2020). Dorsolateral and ventrolateral prefrontal cortex structural changes relative to suicidal ideation in patients with depression. Acta Neuropsychiatr. 32, 84-91. doi: $10.1017 /$ neu.2019.45

Zhou, X., Qi, X. L., and Constantinidis, C. (2016). Distinct roles of the prefrontal and posterior parietal cortices in response inhibition. Cell Rep. 14, 2765-2773. doi: 10.1016/j.celrep.2016.02.072

Conflict of Interest: The authors declare that the research was conducted in the absence of any commercial or financial relationships that could be construed as a potential conflict of interest.

Copyright (c) 2021 Bohaterewicz, Sobczak, Podolak, Wójcik, Mętel, Chrobak, Fafrowicz, Siwek, Dudek and Marek. This is an open-access article distributed under the terms of the Creative Commons Attribution License (CC BY). The use, distribution or reproduction in other forums is permitted, provided the original author(s) and the copyright owner(s) are credited and that the original publication in this journal is cited, in accordance with accepted academic practice. No use, distribution or reproduction is permitted which does not comply with these terms. 